- Original Article

\title{
Short-Term Success Rates of Smoking Cessation Support Programs and Factors Predicting Smoking Relapse: Using Data from a Smoking Cessation Clinic in a Hospital
}

\author{
Seung-Hyun Yu, Myeong-Jun Kim, Jin-Jeon, Hoon-Ki Park*, Hwan-Sik Hwang, Kye-Yeung Park \\ Department of Family Medicine, Hanyang University Medical Center, Seoul, Korea
}

\section{See editorial commentary page on 351}

Background: Although the number of medical institutions running a smoking cessation clinic is on the rise, there remains a paucity of research on the long- and short-term success rates of smoking cessation programs, as well as on smoking relapse rates, before and after project implementation. This study assessed the general characteristics of patients visiting the smoking cessation clinic, success rate of smoking cessation in the short term, and risks of relapse.

Methods: Medical records from March 2015 to April 2017 were analyzed and telephone surveys were conducted with 151 smokers who visited a hospital smoking cessation clinic from March 2015 to April 2017.

Results: Of the 139 smokers who were eligible for follow-up, 22 (15.8\%) failed to quit smoking initially. The clinic's 6 -month success rate of smoking cessation was $64.83 \%$. Those with higher medication compliance had a lower risk of primary failure (odds ratio, 0.056 ; $95 \%$ confidence interval, $0.005-0.609$ ), whereas those with higher age (hazard ratio $[\mathrm{HR}], 0.128 ; \mathrm{P}=0.0252)$ and a greater number of visits to the clinic $(\mathrm{HR}, 0.274 ; \mathrm{P}=0.0124)$ had a lower risk of relapsing.

Conclusion: The risk of primary failure to quit was higher with low medication compliance, and that of relapsing was higher with lower age and fewer number of clinic visits. Various evaluation and analysis methods can be carried out in the future based on the accumulated data for maintenance of smoking cessation and relapse prevention.

Keywords: Smoking; Smoking Cessation; Abstinence; Recurrence; Varenicline

Received: January 26, 2018, Revised: January 25, 2019, Accepted: January 28, 2019

*Corresponding Author: Hoon-Ki Park https://orcid.org/0000-0002-8242-0943

Tel: +82-2-2290-8738, Fax: +82-2-2281-7279, E-mail: hoonkp@hanyang.ac.kr 


\section{INTRODUCTION}

Smoking is associated with various malignant neoplasms and respiratory diseases, including lung, oral, laryngeal, and pancreatic cancer. It is a cause of cardiovascular and cerebrovascular diseases, as well as pregnancy-related complications, and is remediable as well. ${ }^{1)}$

According to a report by the Health Insurance Policy in 2015, the cost of diseases that are caused by smoking in Korea was 7.11 trillion KRW in 2013, 26.0\% higher than that in 2007. ${ }^{2)}$ Smoking is a global concern as it creates huge socioeconomic costs in the long term.

The Sixth Korea National Health and Nutrition Examination Survey revealed that the smoking rate of adults aged 19 years and over was $22.6 \%$ (39.3\% for males and 5.5\% for females) in 2014; they reported that the figure had dropped compared with the previous year and was steadily declining. ${ }^{3)}$ Nonetheless, compared with the 2016 average daily smoking rate of $18.6 \%$, according to the Organization for Economic Cooperation and Development, the daily smoking rate of Koreans remains high at $20.0 \%$. ${ }^{4}$

In 2005, community health centers (253 centers in 2016) began to serve as smoking cessation clinics, and in 2006, smoking cessation counseling services began to be provided by professional counselors via telephone. However, this service only provided counseling and nicotine replacement treatment, and had difficulty in prescribing antismoking medications for active smoking cessation treatment. The 2015 tobacco tax increase provided financial resources, and subsequently, a program to support smoking cessation was proposed.

Since February 2015, the health insurance support project for smoking cessation has lent assistance for all citizens wishing to receive smoking cessation treatment. This health insurance support project provides six counseling sessions and covers the cost of smoking cessation treatment medications (e.g., varenicline, bupropion) or nicotine supplements (e.g., nicotine patch, gum, tablets) up to 3 times a year. A maximum of 18 consultations per year are available for each smoker, and anti-smoking medications and nicotine supplements can be provided for up to 36 weeks per year. When a smoker completed the program, all of the expenses were refunded and health care products (e.g., massage machine, body fat meter, or mouthwash) were given as incentives. Training for doctors was proposed for more effective smoking cessation treatments, and the Health Insurance Corporation conducted online education on smoking cessation for health care workers. ${ }^{5)}$

Amid this situation, the number of hospitals and clinics operating smoking cessation treatment has increased steadily. However, research on the long- and short-term success rates or the difference in the cessation rate before and after the implementation of the health insurance support project remains limited. In this regard, the present study aimed to evaluate the success of the health insurance support project by identifying the general characteristics of patients visiting a smoking cessation clinic at a university hospital in Seoul, the shortterm (3, 6, and 9 months) smoking cessation rate, and the risk of relapse, to help develop effective smoking cessation strategies.

\section{METHODS}

\section{Research Participants}

The health insurance support project for smoking cessation treatment was implemented on February 25, 2015. The study retrospectively investigated 151 smokers who visited and registered in the smoking cessation clinic operated at a hospital from March 2015 to April 2017.

\section{Research Method}

The participants were those who voluntarily registered for smoking cessation treatment, or were sent from other departments, and agreed to enroll in the health insurance support project. They were asked to complete a medical examination questionnaire for smoking cessation treatment at the time of initial registration. This tool included basic personal information, smoking status, history of disease, alcohol intake, nicotine supplement withdrawal symptoms, and nicotine dependence assessment (Korean version of the Fagerstrom Test for Nicotine Dependence, FTND-K).

After completing the medical examination questionnaire, the smokers consulted with a doctor for smoking cessation, and medication was prescribed if needed. Specialized medical doctors or resident physicians in family medicine who had completed the professional education program and qualified for providing smoking cessation treatment were selected as the doctors for smoking cessation counseling. At the time of consultation, the doctors evaluated the smoking cessation decision stage and level of nicotine addiction according to the smoking cessation treatment guidelines of the Agency of Health Care Research and Policy. They also carried out individualized behavioral therapy and pharmacotherapy suitable for each stage. They prescribed varenicline and/or bupropion, and asked and recorded the smokers' smoking status, withdrawal symptoms, drug side effects, difficulty experienced during smoking cessation, confidence in smoking cessation, in accordance with the treatment receivers' circumstances.

For the analysis, we used medical examination questionnaires and retrospective medical records, such as initial records of outpatients, prescription records, and consultation or survey results at the time of the investigation. The investigation was conducted at least 12 weeks after the last smoker enrollment, from July to September 2017. For the smokers who visited during the investigation period, smoking status and medication compliance were investigated at the time of visit. For those who finished their treatment or did not come for the next treatment, smoking status and medication compliance were investigated through telephone survey.

The participants were divided into age groups: 30 years old or younger, 31 to 63 years old, and 64 years old or older. The number of visits for treatment was divided into $1-3,4-6$, and 7 or more visits. The physicians were likewise divided into four groups of three specialists and one resident physician. In terms of alcohol intake, the smokers were asked whether they drink alcohol or not. The visiting route was divided into the voluntary and the requested (by another department) groups. Nicotine dependence was classified as scores of $0-3,4-6$, and 
7-10 on the FTND-K. Comorbidities were recorded based on the records of the first visit. Medication compliance was assessed in the varenicline prescribed group, and was classified into "good" (took all/almost all), "fair" (took half), "poor" (took very little), or "unknown" (no answer/unable to know) by asking the participants at the time of the visit or telephone survey. Successful smoking cessation was defined as self-reported smoking cessation for 7 days at the time of assessment (7-day point abstinence). ${ }^{6}$ Smoking relapse was defined as smoking again after having quit smoking.

\section{Statistical Analyses}

The rate of primary failure and quitting smoking for each variable was compared using t-test and $\chi^{2}$ test. Using logistic regression analysis, a multivariate analysis was performed on age, sex, number of visits, each medical practitioner, alcohol intake, referral source, nicotine dependence, medication compliance, and comorbidities. The odds ratio (OR) related to primary failure and hazard ratio (HR) related to smoking relapse were calculated by Cox proportional hazards regression. We included the time to relapse on survival curves using the KaplanMeier survival analysis for each variable. The monthly success rate of

Table 1. Baseline characteristics of the study subjects

\begin{tabular}{|c|c|c|c|}
\hline Characteristic & $\begin{array}{l}\text { Primary failure } \\
\qquad(n=22)\end{array}$ & $\begin{array}{l}\text { Ever quitter } \\
\quad(n=117)\end{array}$ & P-value \\
\hline Age (y) & $46.8 \pm 15.6$ & $50.0 \pm 13.1$ & 0.31 \\
\hline$<30$ & $5(22.7)$ & $9(7.7)$ & 0.10 \\
\hline $30-63$ & 15 (68.2) & 95 (81.2) & \\
\hline$\geq 64$ & $2(9.1)$ & $13(11.1)$ & \\
\hline Sex (male) & 21 (95.5) & 108 (92.3) & 0.60 \\
\hline No. of visits & $4.64 \pm 7.71$ & $5.82 \pm 5.94$ & 0.50 \\
\hline $1-3$ & $14(63.6)$ & $43(36.8)$ & 0.06 \\
\hline $4-6$ & $5(22.7)$ & $40(34.2)$ & \\
\hline$\geq 7$ & $3(13.6)$ & $34(29.1)$ & \\
\hline \multicolumn{4}{|l|}{ Physician } \\
\hline DR 1 & $9(40.9)$ & $47(40.2)$ & 0.80 \\
\hline DR 2 & 4 (18.2) & $32(27.4)$ & \\
\hline DR 3 & $2(9.1)$ & $9(7.7)$ & \\
\hline Resident & 7 (31.8) & $29(24.8)$ & \\
\hline Alcohol consumption & $13(65.0)$ & $86(74.1)$ & 0.40 \\
\hline \multicolumn{4}{|l|}{ Route of visit } \\
\hline Self-referral & 18 (81.8) & $89(76.1)$ & 0.56 \\
\hline Consultation & 4 (18.2) & $28(23.9)$ & \\
\hline FTND-K* score & $5.43 \pm 2.23$ & $5.05 \pm 2.63$ & 0.54 \\
\hline $0-3$ & 5 (22.7) & 31 (26.5) & 0.83 \\
\hline $4-6$ & $8(36.4)$ & 46 (39.3) & \\
\hline $7-10$ & $9(40.9)$ & $40(34.2)$ & \\
\hline \multicolumn{4}{|c|}{ Compliance to varenicline } \\
\hline Good & $1(4.6)$ & $46(39.3)$ & 0.0003 \\
\hline Fair & $4(18.2)$ & $35(29.9)$ & \\
\hline Poor & $6(27.3)$ & $16(13.7)$ & \\
\hline Unknown & $11(50.0)$ & $20(17.1)$ & \\
\hline Comorbidity (yes) & $12(57.1)$ & $54(46.2)$ & 0.35 \\
\hline
\end{tabular}

Values are presented as mean \pm standard deviation or number (\%). $\mathrm{DR}$, doctor

"Korean version of the Fagerstrom Test for Nicotine Dependence. the smoking cessation clinic was calculated as well. SAS ver. 9.4 (SAS Institute Inc., Cary, NC, USA) was used, and statistical significance was defined as a significance level of $\mathrm{P}<0.05$.

\section{RESULTS}

Of the 151 smokers, 12 (7.9\%) were not followed and, thus, excluded. Of the 139 individuals eligible for follow-up, 22 (15.8\%) were classified as primary failure, as they could not quit smoking during the period of the treatment. The number of patients prescribed with varenicline was 147 (97.4\%). The participants included in the survival analysis included 117 patients who had quit smoking (ever quitter; patients who quit smoking at least once during the investigation), and the 22 primary failures were excluded. At the time of the survey, 75 participants had quit smoking and 42 had relapsed.

\section{General Characteristics of the Participants}

Table 1 compares the characteristics of the primary failure and everquitter groups. The mean age of the participants was 46.8 years in the primary failure group and 50.0 years in the ever-quitter group. Al-

Table 2. Multiple logistic regression results of primary failure in quitting smoking on variables among 139 participants

\begin{tabular}{|c|c|c|}
\hline Characteristic & Univariate & Multivariate $^{\star}$ \\
\hline \multicolumn{3}{|l|}{ Age (y) } \\
\hline$<30$ & Referent & Referent \\
\hline $30-63$ & $0.34(0.09-1.24)$ & $0.21(0.04-1.24)$ \\
\hline$\geq 64$ & $0.35(0.05-2.31)$ & $0.20(0.02-2.39)$ \\
\hline Sex (male vs. female) & $0.63(0.08-5.23)$ & $0.45(0.04-4.73)$ \\
\hline \multicolumn{3}{|l|}{ No. of visits } \\
\hline $1-3$ & Referent & Referent \\
\hline $4-6$ & $0.33(0.10-1.10)$ & $1.69(0.25-11.36)$ \\
\hline$\geq 7$ & $0.30(0.08-1.14)$ & $0.93(0.01-6.84)$ \\
\hline \multicolumn{3}{|l|}{ Physician } \\
\hline Resident & Referent & Referent \\
\hline DR 1 & $1.14(0.35-3.72)$ & $1.74(0.36-8.44)$ \\
\hline DR 2 & $0.73(0.18-2.96)$ & $1.60(0.27-9.48)$ \\
\hline DR 3 & $1.29(0.21-7.82)$ & $2.04(0.19-22.27)$ \\
\hline Alcohol consumption & $0.65(0.24-1.78)$ & $0.42(0.11-1.63)$ \\
\hline \multicolumn{3}{|l|}{ Route of visit } \\
\hline Self-referral & Referent & Referent \\
\hline Consultation & $0.56(0.15-2.03)$ & $0.25(0.05-1.23)$ \\
\hline \multicolumn{3}{|l|}{ FTND-K score } \\
\hline $0-3$ & Referent & Referent \\
\hline $4-6$ & $1.30(0.36-4.72)$ & $1.46(0.32-6.70)$ \\
\hline $7-10$ & $1.50(0.41-5.45)$ & $1.54(0.33-7.06)$ \\
\hline \multicolumn{3}{|l|}{ Compliance to varenicline } \\
\hline Poor & Referent & Referent \\
\hline Fair & $0.38(0.09-1.59)$ & $0.48(0.10-2.40)$ \\
\hline Good & $0.07(0.01-0.64)$ & $0.06(0.01-0.61)$ \\
\hline Unknown & $1.60(0.45-5.63)$ & $2.94(0.42-20.55)$ \\
\hline Comorbidity (yes vs. no) & $1.45(0.56-3.77)$ & $1.79(0.51-6.34)$ \\
\hline
\end{tabular}

Values are presented as odds ratio (95\% confidence interval).

DR, doctor; FTND-K, Korean version of the Fagerstrom Test for Nicotine Dependence. Adjusted for age, sex, number of visits, physician type, alcohol intake, route of visit, FTND-K score, compliance to varenicline, and comorbidity. 
though the ever-quitter group tended to be older, the age difference was not significant $(\mathrm{P}=0.3064)$. The mean number of visits was 4.64 and 5.82 for these groups, respectively. Similarly, the difference was not statistically significant $(\mathrm{P}=0.5003)$. In addition, the ratio of voluntary visits between the primary failure and ever-quitter groups was $81.8 \%$ and $76.1 \%$, respectively; the difference was not significant $(\mathrm{P}=0.5567)$. Moreover, no significant differences were found between the two groups in terms of sex, each medical practitioner, alcohol intake, FTND-K score, and presence of comorbidities. However, the rate of respondents who answered "good" in terms of medication compliance with varenicline was $4.6 \%$ and $39.3 \%$ in the primary failure and ever-quitter groups, respectively $(\mathrm{P}=0.0003)$, indicating that medication compliance was significantly lower in the primary failure group.

\section{Multivariate Analysis (Predictive Factors of Primary Failure and Smoking Relapse)}

A logistic regression analysis was conducted on all 139 participants to calculate the OR associated with the primary failure for each variable (Table 2). The multivariate analysis results showed that participants

Table 3. Hazard ratio of smoking relapse among 117 participants followed up

\begin{tabular}{|c|c|c|c|c|}
\hline \multirow{2}{*}{ Characteristic } & \multicolumn{2}{|c|}{ Univariate } & \multicolumn{2}{|c|}{ Multivariate* } \\
\hline & $\mathrm{HR}$ & P-value & $\mathrm{HR}$ & P-value \\
\hline \multicolumn{5}{|l|}{ Age $(\mathrm{y})$} \\
\hline$<30$ & Referent & & Referent & \\
\hline $30-63$ & 0.41 & 0.05 & 0.29 & 0.02 \\
\hline$\geq 64$ & 0.15 & 0.02 & 0.13 & 0.03 \\
\hline Sex (male vs. female) & 0.79 & 0.74 & 0.89 & 0.88 \\
\hline \multicolumn{5}{|l|}{ No. of visits } \\
\hline $1-3$ & Referent & & Referent & \\
\hline $4-6$ & 0.27 & 0.0009 & 0.27 & 0.01 \\
\hline$\geq 7$ & 0.32 & 0.0041 & 0.30 & 0.02 \\
\hline \multicolumn{5}{|l|}{ Physician } \\
\hline Resident & Referent & & Referent & \\
\hline DR 1 & 0.70 & 0.34 & 0.66 & 0.33 \\
\hline DR 2 & 0.61 & 0.24 & 0.44 & 0.10 \\
\hline DR 3 & 0.40 & 0.22 & 0.25 & 0.10 \\
\hline Alcohol consumption & 1.00 & 0.99 & 0.88 & 0.77 \\
\hline \multicolumn{5}{|l|}{ Route of visit } \\
\hline Self-referral & Referent & & Referent & \\
\hline Consultation & 1.30 & 0.44 & 0.93 & 0.87 \\
\hline \multicolumn{5}{|l|}{ FTND-K score } \\
\hline $0-3$ & Referent & & Referent & \\
\hline $4-6$ & 1.18 & 0.71 & 1.11 & 0.83 \\
\hline $7-10$ & 1.85 & 0.14 & 2.06 & 0.13 \\
\hline \multicolumn{5}{|l|}{ Compliance to varenicline } \\
\hline Poor & Referent & & Referent & \\
\hline Fair & 0.75 & 0.54 & 0.73 & 0.56 \\
\hline Good & 0.41 & 0.07 & 0.45 & 0.13 \\
\hline Unknown & 1.93 & 0.16 & 0.88 & 0.83 \\
\hline Comorbidity (yes vs. no) & 1.02 & 0.96 & 1.10 & 0.80 \\
\hline
\end{tabular}

HR, hazard ratio; DR, doctor; FTND-K, Korean version of the Fagerstrom Test for Nicotine Dependence.

*Adjusted for age, sex, number of visits, physician type, alcohol intake, route of visit, FTND-K score, compliance to varenicline, and morbidity. with "good" compliance to varenicline were less likely to be primary failures compared with the "poor" group (OR, 0.056; 95\% confidence interval [CI], 0.005-0.609). In addition, although the difference was not statistically significant, compared with the group aged younger than 30 years, the OR of the group aged 30 to 63 years was 0.208 (95\% CI, 0.035-1.240), and that of the group over 64 years was 0.201 (95\% CI, 0.017-2.393), indicating that the risk of primary failure decreases with increased age. Further, compared with the group with an FTND-K score of 3 or lower, the OR in the group with a score of 7 or more was 1.535 (95\% CI, 0.334-7.061), indicating that higher nicotine dependence increased the risk of primary failure, though this increase was not statistically significant.

Table 3 shows the risk factors of smoking relapse using Cox analysis of 117 participants (primary failures were excluded). Compared with the group aged younger than 30 years, the HR was $0.289(\mathrm{P}=0.0214)$ in the group aged 30 to 63 years, and $0.128(\mathrm{P}=0.0252)$ in the group above 64 years, indicating that the risk of smoking relapse decreases in older individuals. In addition, compared with the group with 1-3 visits, the HR of smoking relapse was lower in the group with $\geq 7$ or more visits (HR, 0.300; $\mathrm{P}=0.0218$ ), and lowest in the group with 4-6 visits (HR, $0.274 ; \mathrm{P}=0.0124$ ). No statistically significant difference was seen in the risk of smoking relapse in terms of sex, physician, alcohol intake, referral source, nicotine dependence, and presence of comorbidities. In terms of medication compliance, the HR of the "fair" group was 0.734 $(\mathrm{P}=0.5634)$, and that of the "good" group was $0.448(\mathrm{P}=0.1337)$, compared with the "poor" group. The HR tended to decrease gradually, but not to statistically significant degree.

\section{Survival Analysis}

Table 4 and Figure 1 show the monthly success rate of the smoking cessation clinic using the Kaplan-Meier plot. The success rate was $73.67 \%$ at 3 months, $64.83 \%$ at 6 months, and $61.57 \%$ at 9 months. When comparing the success rate of smoking cessation by variable,

Table 4. Table for monthly survival (quitting smoking) rate of the smoking cessation clinic by using Kaplan-Meier plot

\begin{tabular}{rrrcc}
\hline Months & Failed & Censored & $\begin{array}{c}\text { Survival } \\
\text { (quitting) }\end{array}$ & Standard error \\
\hline 1 & 17 & 3 & 1 & 0 \\
2 & 13 & 3 & 0.85 & 0.03 \\
3 & 4 & 2 & 0.74 & 0.04 \\
4 & 3 & 8 & 0.70 & 0.04 \\
5 & 2 & 6 & 0.67 & 0.04 \\
6 & 0 & 4 & 0.65 & 0.05 \\
7 & 1 & 13 & 0.65 & 0.05 \\
8 & 1 & 7 & 0.63 & 0.05 \\
9 & 0 & 9 & 0.62 & 0.05 \\
10 & 1 & 5 & 0.62 & 0.05 \\
11 & 0 & 6 & 0.58 & 0.06 \\
12 & 0 & 2 & 0.58 & 0.06 \\
13 & 0 & 2 & 0.58 & 0.06 \\
15 & 0 & 0 & 0.58 & 0.06 \\
$\ldots$ & 0 & 5 & 0.58 & 0.06 \\
\hline
\end{tabular}




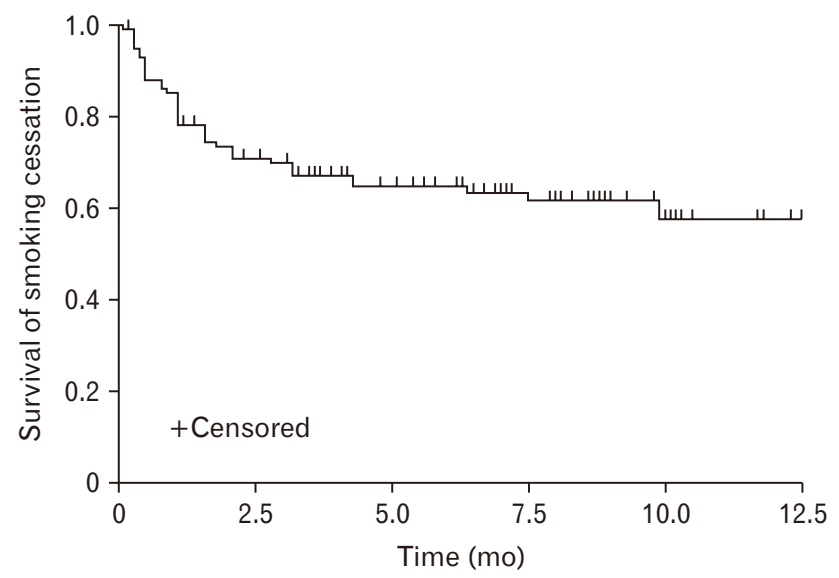

Figure 1. Survival curve for smoking cessation.

significant differences were found for the number of visits $(\mathrm{A}, \mathrm{P}=$ 0.0003), age ( $\mathrm{B}, \mathrm{P}=0.0251)$, and medication compliance $(\mathrm{C}, \mathrm{P}=0.0011)$. There was no significant difference in the success rate of smoking cessation according to nicotine dependence ( $\mathrm{D}, \mathrm{P}=0.2795)$ or physician (E, $\mathrm{P}=0.4669$ ) (Figure 2).

\section{DISCUSSION}

The present study aimed to investigate the predictive factors for smoking cessation treatment results and smoking cessation success after implementation of the health insurance support project.

The clinic's 6-month smoking cessation rate was $64.8 \%$, which was shown to be higher than the Community Health Center's existing smoking cessation project result (44.8\%). This difference may have been caused by the change in smoking cessation treatment, from providing smoking cessation counseling and supplements to the current smoking cessation treatment project, which includes professional intervention and drug treatment by a physician.

Studies have reported that continuous smoking cessation treatment through insurance support increases the success rate of smoking cessation. In Turkey, a smoking cessation treatment support program was implemented in 2008 as a part of a national plan aimed at reducing the smoking rate. The smoking rate of adults declined from $31.2 \%$ to $27.1 \%$ over the 5 years after the implementation of the program. ${ }^{7)}$ In the United States, a number of states have seen a considerable decrease in smoking rates, from $38.3 \%$ in 2006 to $28.3 \%$ in 2008 . $^{8)}$ Japan has also supported insurance payments for smoking cessation treatment, starting in 2006, and succeeded in reducing the smoking rate from $24.2 \%$ in 2005 to $19.5 \%$ in $2010 .{ }^{9)}$ Currently, the Korean health insurance support project allows individuals to receive treatment for smoking cessation without shouldering costs, by supporting medical and drug expenses for 12 weeks or six visits up to 3 times a year. This reduction in economic burden may have increased access to smoking cessation treatment, which in turn may have increased the rates of smoking cessation.
Reports have pointed to a trend in treatment recipients in which the higher the age, the higher the success rate of smoking cessation. According to Tait et al., ${ }^{10)}$ elderly patients seeking smoking cessation treatment are often more likely to be confronted with health-related problems that are caused by smoking and are more motivated to quit smoking owing to the more immediate health advantages associated with quitting smoking. Our findings were consistent with this trend; the risk of smoking relapse decreased with age.

In this study, the concept of primary failure was used to compare with quitting smoking at any point during treatment. "Primary failure" referred to the group that could not quit smoking during the period of receiving smoking cessation treatment; 22 (15.8\%) of 139 participants who were eligible for follow-up belonged to this group. Nine of them were unable to be contacted after their first visit. The remaining 13 had at least three visits to the clinic; they reported that smoking desire had decreased compared with before the treatment, and that their actual amount of smoking had been greatly reduced although they could not quit smoking. Many of them cited the side effects of varenicline, such as nausea and abdominal discomfort, as the reasons for their failure to quit smoking. Compared with a nicotine substitute or sustained-releasing bupropion, which have been used for smoking cessation, varenicline is a drug that has shown a superior smoking cessation effect ${ }^{11)}$ and a capacity-dependent smoking cessation effect. ${ }^{12,13)}$ In other words, the longer the medication is used, the greater the success rate for smoking cessation. In our study, the group with "good" medication compliance had fewer primary failures and lower incidence of relapse. In addition, given that the success rate of smoking cessation was higher when the number of visits to the clinic was greater, then frequent visits to the doctor's office to check the status of smoking and medication served as important factors. Notably, the frequent side effects of varenicline include nausea, vomiting, and sleep disturbance. ${ }^{14)}$ As there is a possibility of patients stopping the medication owing to unexpected drug side effects, physicians should follow-up with patients often and closely examine their condition and adjustment.

The physicians included in this smoking cessation treatment project were those who had completed the specialist education on smoking cessation provided by the Health Insurance Corporation. They provide appropriate counseling and pharmacotherapy according to the patient's smoking cessation stage. In this study, the risk of primary failure or smoking relapse was not significantly different between the group treated by a specialist and that treated by a resident physician. In the practice of smoking cessation treatment, specialists would have used in-depth interview techniques based on more abundant medical knowledge and treatment experience compared with residents. Studies have reported that appropriate motivational interviewing contributes significantly to the success of smoking cessation. ${ }^{15)}$ However, in the present work, regular visits to the clinic on the scheduled day of treatment, along with medication compliance, were more important factors. Smoking cessation physicians should educate patients on the importance of visiting the clinic as scheduled and conduct interviews centered on strategies for stimulating motivation for smoking cessa- 

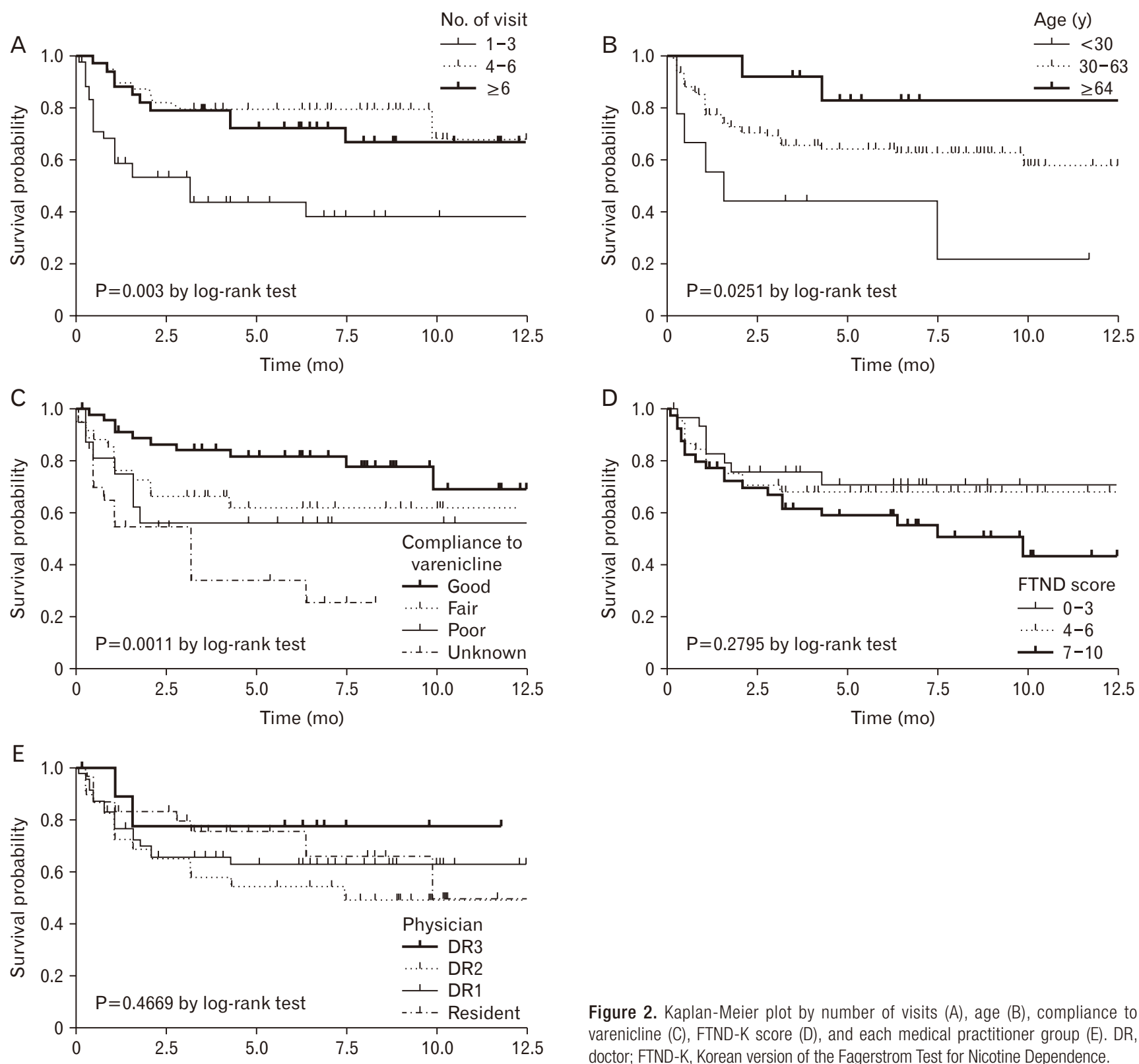

Figure 2. Kaplan-Meier plot by number of visits (A), age (B), compliance to varenicline (C), FTND-K score (D), and each medical practitioner group (E). DR, doctor; FTND-K, Korean version of the Fagerstrom Test for Nicotine Dependence.

tion and preventing relapse.

The limitations of this study are that, first, a self-report survey was used; thus, the smoking cessation maintenance condition might have been reported as higher than it actually was. Recall bias could not be avoided as the study relied on medical examination records and telephone questionnaires. However, a study conducted on 294 smokers who attended a smoking cessation clinic showed a high agreement between the self-reported smoking status and urine cotinine data for over 4 weeks (kappa coefficient=0.79; 95\% CI, 0.70-0.88). ${ }^{16)}$ Moreover, the accuracy of self-reported smoking status has been reported to be higher than $95 \%$ in a number of previous studies. ${ }^{16)}$ Second, it was not possible to evaluate long-term success rates in smoking cessation as this study investigated patients at one hospital in one city over a rela-

tively short period of time. Although the analysis results are meaningful, the small size of our sample may have limited the ability to find statistically significant results. Studies have found that the combination of treatment using varenicline and counseling leads to increases in long-term cessation success rates over more than 2 years; it is likely that future evaluations may also find similar meaningful results in terms of long-term smoking cessation. Third, this study was an observational one without a control group and could not provide comparative results on the effectiveness of the smoking cessation support program.

Nevertheless, this study's relevance is that it is one of the first to evaluate factors related to the maintenance of smoking cessation and smoking relapse, after the implementation of the smoking cessation 
health insurance support project. Our results suggest that, in terms of the risk of primary failure with low medication complication, the risk of relapse tends to be higher in younger smokers and in those who missed clinic visits. Therefore, care should be taken with regard to these factors during smoking cessation treatment. Systematic and comprehensive evaluation and analysis, based on the accumulated data, are needed, to establish a concrete action plan for a smoking cessation policy aimed at reducing smoking rates in Korea.

\section{CONFLICT OF INTEREST}

No potential conflict of interest relevant to this article was reported.

\section{ORCID}

Seung-Hyun Yu: https://orcid.org/0000-0003-3694-521X

Myeong-Jun Kim: https://orcid.org/0000-0002-3516-6698

Jin-Jeon: https://orcid.org/0000-0001-6446-9578

Hoon-Ki Park: https://orcid.org/0000-0002-8242-0943

Hwan-Sik Hwang: https://orcid.org/0000-0002-6248-7411

Kye-Yeung Park: https://orcid.org/0000-0003-4512-5686

\section{REFERENCES}

1. Burns DM. Nicotine addiction. In: Wiener CM, Kasper DL, Braunwald E, Fauci AS, Hauser SL, Longo DL, et al. editors. Harrison's principles of internal medicine. 19th ed. New York (NY): McGraw-Hill; 2005. p. 2729-32.

2. Health Insurance Policy Research Institute. Assessing socio-economic effects of major health risk factors and the effects of regulatory policy [Internet]. Seoul: National Health Insurance Service; 2015 [cited 2018 Jun 22]. Available from: http://www.nhis.or.kr/menu/boardRetriveMenuSet.xx?menuId=F3430.

3. Korea Centers for Disease Control and Prevention. The Sixth Korea National Health and Nutrition Examination Survey (KNHANES VI-2), 2014 [Internet]. Cheongju: Korea Centers for Disease Control and Prevention; c2014 [cited 2018 Jun 22]. Available from: http://cdc.go.kr.

4. Korea Statistics Office. Smoking rate (OECD) [Internet]. Daejeon: Statistics Korea; 2019 [cited 2018 Jun 22]. Available from: http://kosis.kr/
statHtml/statHtml.do?orgId=101\&tblId=DT_2KAAC04_OECD\# (Korean).

5. Paek YJ, Choi JK, Lee ES, Jo MW. Smoking cessation services provided by the National Health Insurance Service. J Korean Med Assoc 2018; 61:157-62.

6. Velicer WF, Prochaska JO. A comparison of four self-report smoking cessation outcome measures. Addict Behav 2004;29:51-60.

7. Celik I, Yuce D, Hayran M, Erman M, Kilickap S, Buzgan T, et al. Nationwide Smoking Cessation Treatment Support Program: Turkey project. Health Policy 2015;119:50-6.

8. Land T, Warner D, Paskowsky M, Cammaerts A, Wetherell L, Kaufmann R, et al. Medicaid coverage for tobacco dependence treatments in Massachusetts and associated decreases in smoking prevalence. PLoS One 2010;5:e9770.

9. Kumiko S, Tomotaka S, Masakazu N, Akira O, Keiji W, Nobuyuki H, et al. Smoking prevalence and beliefs on smoking cessation among members of the Japanese Cancer Association in 2006 and 2010. Cancer Sci 2012;103:1595-9.

10. Tait RJ, Hulse GK, Waterreus A, Flicker L, Lautenschlager NT, Jamrozik $\mathrm{K}$, et al. Effectiveness of a smoking cessation intervention in older adults. Addiction 2007;102:148-55.

11. Jorenby DE, Hays JT, Rigotti NA, Azoulay S, Watsky EJ, Williams KE, et al. Efficacy of varenicline, an alpha4beta2 nicotinic acetylcholine receptor partial agonist, vs placebo or sustained-release bupropion for smoking cessation: a randomized controlled trial. JAMA 2006;296:5663.

12. Nakamura M, Oshima A, Fujimoto $Y$, Maruyama N, Ishibashi T, Reeves KR. Efficacy and tolerability of varenicline, an alpha4beta2 nicotinic acetylcholine receptor partial agonist, in a 12-week, randomized, placebo-controlled, dose-response study with 40-week follow-up for smoking cessation in Japanese smokers. Clin Ther 2007;29:1040-56.

13. Lee JH, Jones PG, Bybee K, O'Keefe JH. A longer course of varenicline therapy improves smoking cessation rates. Prev Cardiol 2008;11:210-4.

14. Garrison GD, Dugan SE. Varenicline: a first-line treatment option for smoking cessation. Clin Ther 2009;31:463-91.

15. Lindson-Hawley N, Thompson TP, Begh R. Motivational interviewing for smoking cessation. Cochrane Database Syst Rev 2015;(3):CD006936.

16. Park SS, Lee JY, Cho SI. Validity of expired carbon monoxide and urine cotinine using dipstick method to assess smoking status. J Prev Med Public Health 2007;40:297-304. 